\title{
Correction to: Graph Matching Based Connectomic Biomarker with Learning for Brain Disorders
}

Rui Sherry Shen, Jacob A. Alappatt, Drew Parker, Junghoon Kim,

Ragini Verma, and Yusuf Osmanlıŏlu

\section{Correction to: \\ Chapter "Graph Matching Based Connectomic Biomarker with Learning for Brain Disorders" in: C. H. Sudre et al. (Eds.): Uncertainty for Safe Utilization of Machine Learning in Medical Imaging, and Graphs in Biomedical Image Analysis, LNCS 12443, https://doi.org/10.1007/978-3-030-60365-6_13}

The original version of this chapter was revised. Figure 2 was updated with the correct numbers.

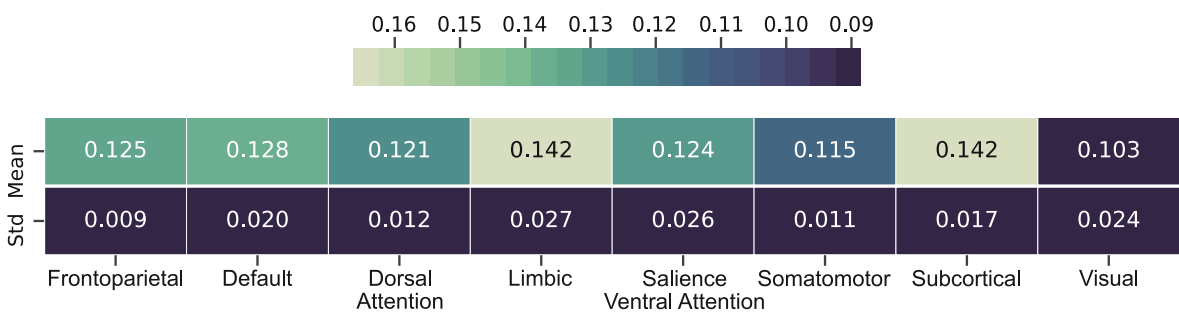

Fig. 2. Dysfunction coefficients at functional systems level, with larger dysfunction coefficients indicating a dominant pathology effect at the associated functional systems. We observe large coefficient values for limbic and subcortical networks, which highlights their vulnerability to injury. Note that, dysfunction coefficients are normalized by the total sum of coefficients to show the relative vulnerability of the systems.

The updated version of this chapter can be found at https://doi.org/10.1007/978-3-030-60365-6_13 